optical properties, which allowed us to use a specialized microscope tuned to the near-infrared to pinpoint their locations within the cells."

Weisman, Paul Cherukuri of the Texas Heart Institute, and their colleagues reported their research in the December 8, 2004, issue of the Journal of the American Chemical Society (p. 15638; doi: 10.1021/ja0466311). The researchers cultured mouse macrophage cells in solutions containing between 0 ppm and 7 ppm single-walled carbon nanotubes (SWNTs) for periods of up to $96 \mathrm{~h}$. They found that the amount of SWNTs taken up by the cells increased smoothly as the concentration or the time of exposure increased. In addition, some cells cultured at cooler temperatures showed a slower rate of uptake, a finding that suggested that the nanotubes were being ingested through normal phagocytosis.

The samples were studied using a spectrofluorometer and a fluorescence microscope that was modified for near-infrared imaging by the addition of a digital camera containing InGaAs detector elements.

Not only did the nanotubes retain their optical signatures after entering the white blood cells, but the introduction of nanotubes caused no measurable change in cell properties like shape, rate of growth, or the ability to adhere to surfaces.

The new findings demonstrate that SWNTs might be valuable biological imaging agents, in part because SWNTs fluoresce in the near-infrared portion of the spectrum, at wavelengths not normally emitted by biological tissues. This may allow light from even a handful of nanotubes to be selectively detected from within the body. This option may prove particularly beneficial in cases where the bleaching, toxicity, and degradation of more traditional markers are problematic.

\section{Copper Oxide Nanotube Arrays Produced by MOCVD Process with Template}

Because of the tremendous improvement in chemical properties resulting from the incorporation of nanostructured materials, inorganic nanostructures have been extensively studied for applications such as electrical contacts, catalytic gassensing devices, and solar cells. Various techniques, including metalorganic chemical vapor deposition (MOCVD), have been attempted for synthesizing inorganic (metal, oxide, halide, and chalcogenide) nanotubes or nanowires. Researchers are now trying to achieve the synthesis of $\mathrm{CuO}$ nanotubes by a simple and reproducible method using a so-called "template" to help create well-aligned nanostructures, which may have applica- tions in devices containing copper oxide thin films or as cathode materials in lithium copper oxide electrochemical cells. In the December 28, 2004, issue of Chemistry of Materials (p. 5559; doi: 10.1021/cm048685f), researchers from Università di Catania in Italy announced that they have grown homogeneous $\mathrm{CuO}$ nanotube arrays on a template by MOCVD.

G. Malandrino of Università di Catania and colleagues used anodic aluminum oxide membranes as a template. The 60- $\mu$ m-thick template consists of a precise, non-deformable structure, with pores $200 \mathrm{~nm}$ in diameter arranged in a
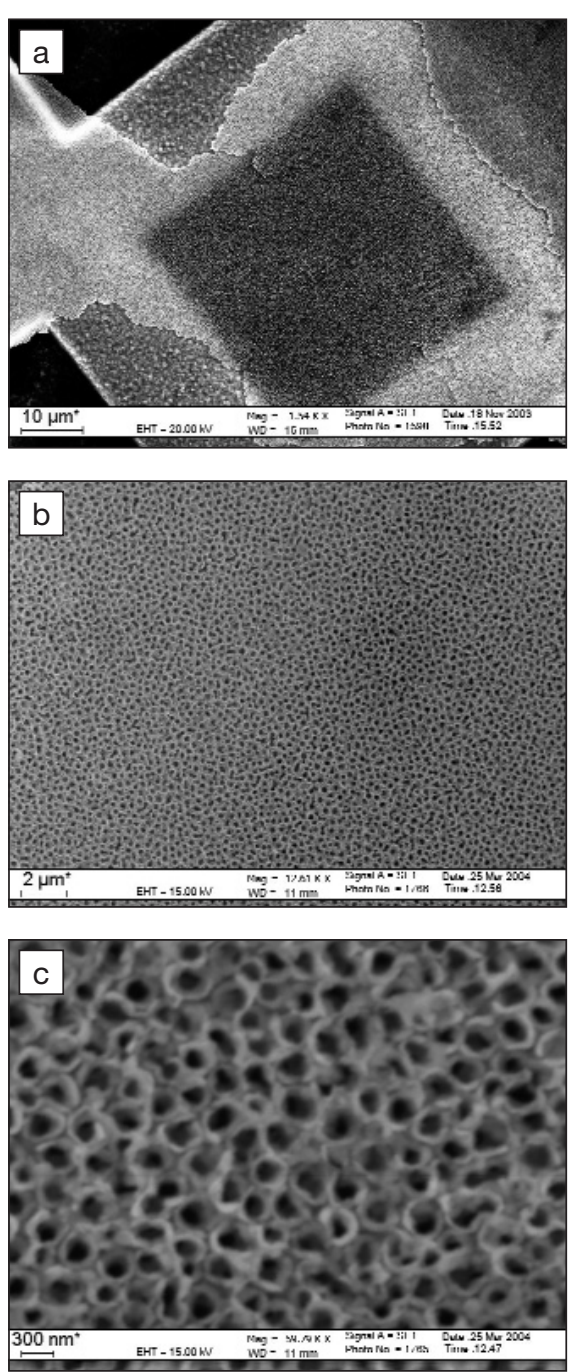

Figure 1. Freestanding CuO nanotube arrays: (a), (b) low-magnification planview scanning electron microscopy images of the $\mathrm{CuO}$ nanotube sheet; and (c) high-magnification plan view of the nanotube sheet. Reprinted with permission from Chemistry of Materials 16 (26) (2004) p. 5559. (C) 2004 American Chemical Society. hexagonal array. The membrane was positioned vertically in a hot-wall reactor and maintained at $400^{\circ} \mathrm{C}$. Oxygen and argon were used as the reaction gas and carrier gas, respectively. The $\mathrm{Cu}(\mathrm{tmhd})_{2}$ (Htmhd = 2,6,6,6-tetramethyl-3,5-heptandione) source was sublimed at $100^{\circ} \mathrm{C}$. The deposition was carried out for $3 \mathrm{~h}$ at a total pressure of 2 Torr.

Scanning electron micrographs show that the outer diameters of the $\mathrm{CuO}$ nanotubes are $\sim 250 \mathrm{~nm}$ and the wall thicknesses are $\sim 40 \mathrm{~nm}$. The nanotubes are $\sim 1 \mu \mathrm{m}$ long. The researchers said that the characteristic pore dimensions of the template membrane influence the diameters of the nanotubes, whereas the length of the nanotubes depends on the process conditions. Furthermore, according to the researchers, the template can be completely removed by dissolving it in a $\mathrm{NaOH}$ solution, resulting in freestanding $\mathrm{CuO}$ nanotube arrays (see Figure 1).

"To our knowledge, this is the first example of a regularly packed $\mathrm{CuO}$ nanotube array made through a rather straightforward process," said the researchers. The "transfer" of a well-assessed MOCVD procedure for the deposition of $\mathrm{CuO}$ films to analogue nanotubes, they assert, could open viable routes to the fabrication of nanotube arrays of various oxides.

SHIMING WU

\section{Silicalite-1 Self-Supported Micromembrane Separates Propane/Nitrogen Gas Mixture}

Zeolite membranes are used industrially as an aid during the pervaporation process for the dehydration of solvents. They are currently under development for applications such as the separation of certain gas/hydrocarbon mixtures, membrane reactors, and sensors, and heading toward applications in silicon-based microdevices. Among several constraints to take into consideration are the loss of separation selectivity at high temperatures and the high cost of the membranes, at more than $\$ 2600$ per $\mathrm{m}^{2}$. Efforts are concentrated on developing small-scale membranes, which could potentially be single crystals with improved stability during fabrication and thermal cycling. Following this direction, E. Mateo and colleagues from the University of Zaragoza, Spain, have created arrays of micromembranes of silicalite- 1 on a stainless steel sheet, which they reported in the November 30, 2004, issue of Chemistry of Materials (p. 4847; doi: $10.1021 / \mathrm{cm} 048504+$ ).

The investigators fabricated the silicalite-1 powders for seeding, starting from a solution of tetrapropylammonium hydroxide (TPAOH), tetraethyl orthosili- 
cate (TEOS), water, and ethanol mixed with molar ratios of $9 / 25 / 408 / 100$. Subsequent hydrothermal synthesis at $100^{\circ} \mathrm{C}$ for $18 \mathrm{~h}$ resulted in a gel, which was aged for $24 \mathrm{~h}$ and later calcined at $480^{\circ} \mathrm{C}$ for $8 \mathrm{~h}$. Particles thus obtained were $100 \mathrm{~nm}$ in diameter, and x-ray diffraction (XRD) revealed that the only crystalline phase present was silicalite-1.

The round stainless steel sheet used as a substrate was $75 \mu \mathrm{m}$ thick and $15 \mathrm{~mm}$ in diameter and had laser-drilled holes of about $80 \mu \mathrm{m}$ in diameter. These holes, drilled by a Nd:YAG laser in pulse mode, were in a square lattice pattern a little over $200 \mu \mathrm{m}$ apart. The pulse-drilling technique left rough edges around each hole on one side of the sheet. The silicalite- 1 nanocrystals already synthesized were rubbed against this side of the substrate and thus filled the holes. The substrate was then placed vertically in a solution of $\mathrm{KOH} /$ tetrapropylammonium bromide (TPABr)/ TEOS $/ \mathrm{H}_{2} \mathrm{O}$ mixed with molar ratios of $1 / 1 / 4.5 / 100$ in an autoclave.

Hydrothermal synthesis at $170^{\circ} \mathrm{C}$ for $24 \mathrm{~h}$ resulted in a $30-\mu \mathrm{m}$-thick membrane of silicalite- 1 crystals on the rough side of the substrate along with the silicalite- 1 that completely filled the holes in the stainless steel sheet. XRD showed silicalite- 1 as the only phase present in the membranes. An array of micromembranes was capable of separating propane from $\mathrm{N}_{2}$, and had a permeance higher than the MFI-type current membranes. This is significant progress, the researchers said, since the membranes are essentially self-supported, with the zeolite in contact with both the feed and the permeate sides through a relatively small thickness.

Changing the temperature or time of the hydrothermal synthesis varies the thickness of the resulting uniform silicalite- 1 layer. When the researchers tried synthesis at $100^{\circ} \mathrm{C}$, the thickness of the uniform silicalite-1 layer was less than $2 \mu \mathrm{m}$, while the perforations were still completely filled with silicalite- 1 . However, the thinner layer resulted in less desirable permeation properties.

SIARI SOSA

\section{Properties of Heavy-Fermion \\ Materials Demystified}

Theoretical physicists Q. Si of Rice University and P. Coleman of Rutgers University, along with a team of experi- mental physicists led by F. Steglich at the Max Planck Institute for Chemical Physics of Solids in Dresden, have shown that the Fermi volume in materials with strongly correlated electrons changes its size abruptly at a "quantum critical point" as the temperature of the material is lowered near absolute zero Kelvin.

"Quantum critical points are of great current interest because of their ability to reach up from absolute zero and create a new state of matter called 'quantum critical matter,"' said Coleman. "This may provide a route to many new classes of material."

The research project, reported in the December 16, 2004, issue of Nature (p. 881; doi:10.1038/nature03129), addressed whether the Fermi surface transformation at the quantum critical point developed gradually, as expected if the magnetism is of spin-density-wave type, or suddenly, as expected if the heavy electrons are abruptly localized by magnetism. Studying the heavy-fermion metal $\mathrm{YbRh}_{2} \mathrm{Si}_{2}$, the researchers analyzed Hall effect data, which revealed an abrupt change in the Fermi surface transformation that the researchers attribute to the breakdown of the Kondo effect.

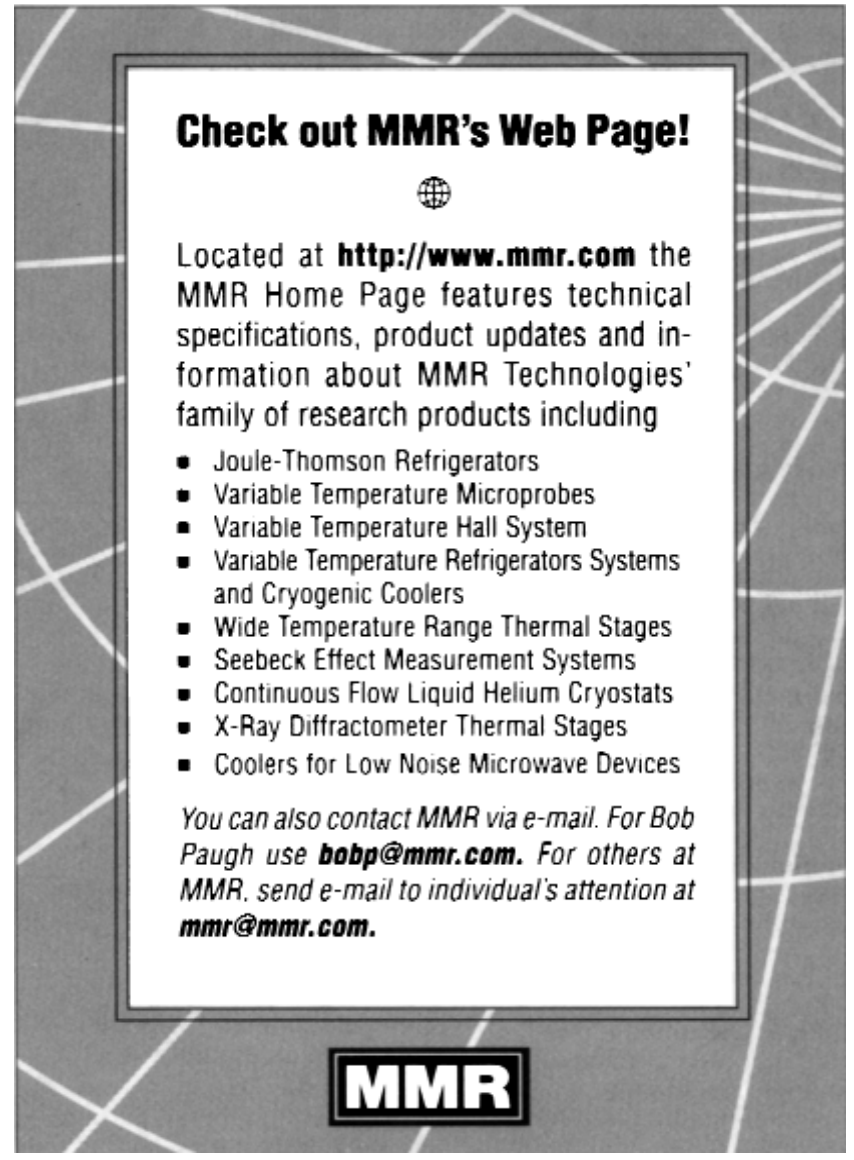

For more information, see http://advertisers.mrs.org

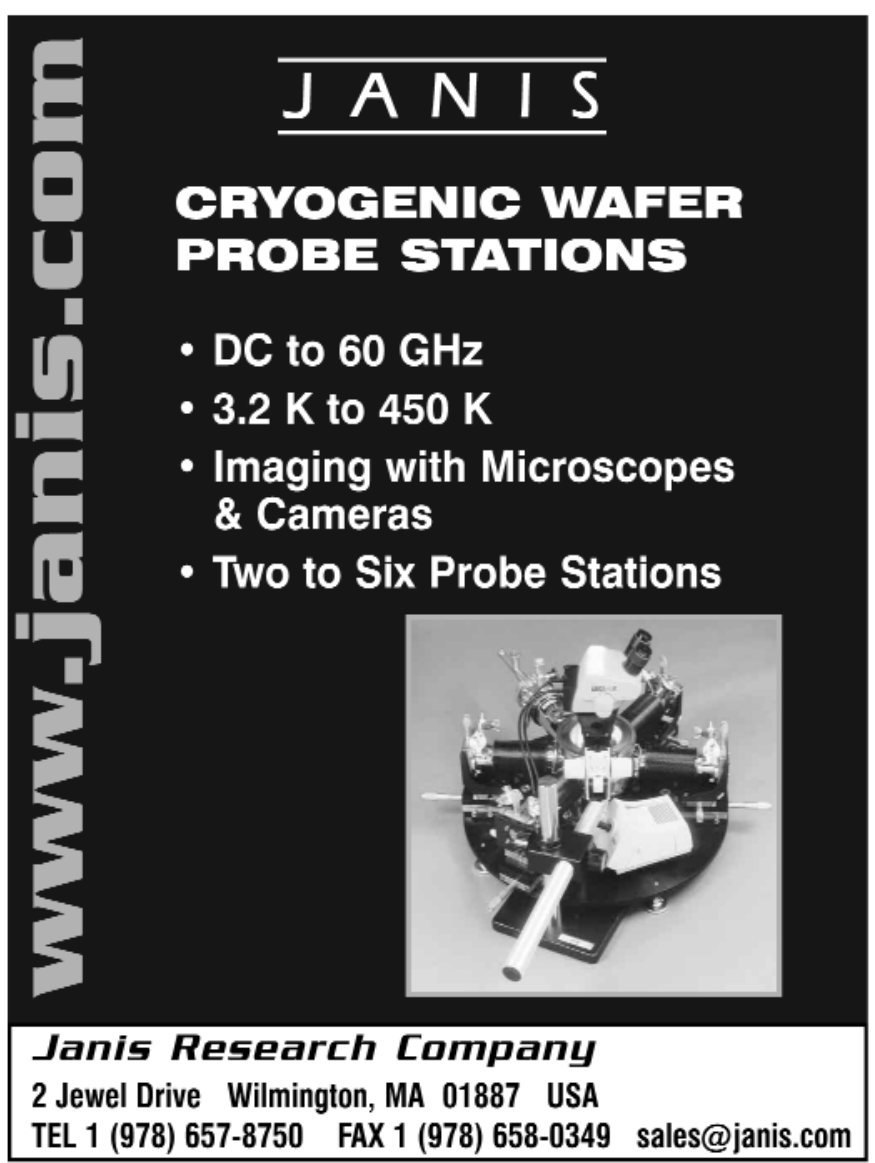

For more information, see http://advertisers.mrs.org 